\title{
Potencial hidrogeniônico de soluções de antibióticos submetidas a condições ambientais: ensaio preliminar
}

\author{
HYDROGEN POTENTIAL OF ANTIBIOTIC SOLUTIONS SUBJECTED TO \\ ENVIRONMENTAL CONDITIONS: A PRELIMINARY TRIAL
}

\section{POTENCIAL DE HIDRÓGENO DE SOLUCIONES DE ANTIBIÓTICOS SOMETIDAS A CONDICIONES AMBIENTALES: ENSAYO PRELIMINAR}

\section{Cintia Monteiro', Renata Maria Coelho Crepaldi ${ }^{2}$, Ariane Ferreira Machado Avelar ${ }^{3}$, Maria Angélica Sorgini Peterlini ${ }^{4}$, Mavilde Da Luz Gonçalves Pedreira ${ }^{5}$}

\section{RESUMO}

Estudo experimental para aferição do potencial hidrogeniônico $(\mathrm{pH})$ dos antimicrobianos ceftriaxona sódica, cloridrato de vancomicina, metronidazol, penicilina $G$ potássica e sulfato de amicacina, após reconstituição, diluição com $\mathrm{NaCl} 0,9 \%$ (SF) e soro glicosado $5 \%$ (SG), em oito momentos distintos e sob condições cotidianas de luminosidade e temperatura ambiente de unidade hospitalar não climatizada. $\mathrm{O}$ objetivo deste estudo foi verificar alterações no comportamento ácido-básico das soluções, indicativas de instabilidade química ou relacionadas a complicações da terapia intravenosa. Nos 186 valores de pH analisados, não foram identificadas variações maiores que 1,0 valor nem alterações físicas visíveis a olho nu. Todas as soluções tiveram $\mathrm{pH}$ menor que 7 e não houve diferença considerável para a prática clínica segundo o diluente. As médias dos valores de $\mathrm{pH}$ após a diluição em SF e SG, do cloridrato de vancomicina, metronidazol e sulfato de amicacina constituem fator de risco para o desenvolvimento de complicações intravenosas devido a sua extrema acidez.

\section{DESCRITORES}

Concentração de Íons de Hidrogênio

Estabilidade de medicamentos

Infusões intravenosas

Erros de medicação

Enfermagem pediátrica

\begin{abstract}
This experimental study was performed to assess the hydrogen potential $(\mathrm{pH})$ of the antimicrobials ceftriaxone sodium, vancomycin hydrochloride, metronidazole, penicillin $\mathrm{G}$ potassium, and amikacin sulphate, following reconstitution, diluted with $\mathrm{NaCl} 0.9 \%$ (SF) and glucose solution 5\% (GS), at eight different time intervals and under the normal daily conditions of lighting and temperature within the hospital unit (no air conditioning). The objective of this study was to verify the changes in the acid-base behavior of the solutions, which indicate chemical instability and can be associated with complications during intravenous therapy. Of the 186 analyzed $\mathrm{pH}$ values, there were no variations greater than 1.0 and no physical alterations visible to the naked eye. All solutions had a pH less than 7 , and there were no significant differences for clinical practice regarding the diluent. The mean $\mathrm{pH}$ values after dilution with SF and GS for vancomycin hydrochloride, metronidazole, and amikacin sulphate are a risk factor for the development of intravenous complications due to their extreme acidity.
\end{abstract}

\author{
DESCRIPTORS \\ Hidrogen-Ion concentration \\ Drug stability \\ Infusions, intravenous \\ Medication errors \\ Pediatric nursing
}

\section{RESUMEN}

Estudio experimental de contraste del potencial de hidrógeno $(\mathrm{pH})$ de antimicrobianos ceftriaxona sódica, clorhidrato de vancomicina, metronidazol, penicilina $G$ potásica y sulfato de amikacina, luego de reconstitución, dilución con $\mathrm{NaCl} 0,9 \%$ (SF) y suero glucosado $5 \%$ (SG), en ocho momentos distintos, bajo condiciones normales de luminosidad y temperatura de unidad hospitalaria no climatizada. Objetivó verificar alteraciones del comportamiento ácido-básico de las soluciones, indicativas de inestabilidades químicas o relacionadas a complicaciones de terapia intravenosa. En $186 \mathrm{pH}$ analizados, no se identificaron variaciones mayores a 1,0 valor, ni alteraciones físicas a simple vista. Todas las soluciones tuvieron $\mathrm{pH}$ menor a 7 , no hubo diferencia considerable para la práctica clínica según el diluyente. Los promedios de valor de pH luego de dilución en SF y SG para clorhidrato de vancomicina, metronidazol y sulfato de amikacina constituyen factor de riesgo para desarrollo de complicaciones intravenosas debido a su extrema acidez.

\section{DESCRIPTORES}

Concentración de lones de Hidrógeno Estabilidad de medicamentos Infusiones intravenosas

Errores de medicación Enfermería pediátrica

\footnotetext{
${ }^{1}$ Graduanda em Enfermagem da Universidade Federal de São Paulo. Bolsista CNPq. São Paulo, SP, Brasil. cintiamonteiromsp@hotmail.com ${ }^{2}$ Enfermeira Graduada pela Universidade Federal de São Paulo. São Paulo, SP, Brasil. recrepaldi@hotmail.com ${ }^{3}$ Professora Adjunta da Disciplina de Enfermagem Pediátrica da Escola Paulista de Enfermagem da Universidade Federal de São Paulo. São Paulo, SP, Brasil. ariane.machado@unifesp.br 4 Professora Adjunta da Disciplina de Enfermagem Pediátrica da Escola Paulista de Enfermagem da Universidade Federal de São Paulo. São Paulo, SP, Brasil. maria. angelica@unifesp.br ${ }^{5}$ Professora Associada da Disciplina de Enfermagem Pediátrica da Escola Paulista de Enfermagem da Universidade Federal de São Paulo. Bolsista de Produtividade em Pesquisa do CNPq. São Paulo, SP, Brasil. mpedreira@unifesp.br
} 


\section{INTRODUÇÃO}

Desde 1860, Florence Nightingale enfatizava a necessidade de enfermeiros conhecerem a correta preparação, dosagem, técnica de administração, efeitos desejados e colaterais de cada medicamento. Em meados de 1950, a enfermagem iniciou suas atribuições na administração de fármacos e soluções pela via intravenosa (IV), utilizada em pacientes com inabilidade de ingesta adequada da quantidade necessária de fluídos, eletrólitos, vitaminas e ou calorias, em situação de desequilíbrio hidroeletrolítico, perda de sangue, disfunção de vários órgãos e sistemas, infecção, procedimentos cirúrgicos e ou grandes queimaduras, dentre outros ${ }^{(1)}$.

Estudo realizado em um hospital universitário constatou que a média de doses de fármacos de administração intravenosa utilizados em crianças por dia foi de 6,8. Tal resultado demonstra o grande emprego dessa terapia nos pacientes pediátricos ${ }^{(2)}$. Entre os fármacos infundidos por via IV destacam-se os antibióticos, que requerem cuidados especializados de enfermagem para a sua administração, referentes à diluição e ao tempo de infusão ${ }^{(3)}$. Devido à falta da apresentação de medicamentos para esta faixa etária no mercado nacional, é frequente a rediluição antes de serem administrados. Porém, questionamentos surgem quanto à estabilidade do fármaco após nova diluição, sua esterilidade, presença de pirógenos e tempo de estocagem até o momento da administração ${ }^{(2)}$.

A manutenção da estabilidade farmacológica e compatibilidade físico-química são elementos críticos na oferta correta e apropriada da terapia medicamentosa para o paciente. A adequação e a segurança da terapia podem ser adversamente afetadas pela instabilidade medicamentosa. O potencial para problemas de estabilidade do medicamento é grande, devido ao longo tempo de contato e exposição às condições do ambiente, como temperatura e luz ${ }^{(4)}$.

A instabilidade medicamentosa é definida como a ocorrência de reações químicas que resultam em mudanças moleculares originando diferentes compostos denominados produto degradado. O produto degradado pode ser um princípio terapêutico inativo ou, até mesmo, uma substância tóxica produzida na reação. Assume-se estabilidade de um fármaco o período no qual o mesmo se mantém com até $90 \%$ da sua concentração inicial, seja em sua apresentação, reconstituição ou diluição. Uma solução medicamentosa estável apresenta moléculas com eletrosferas que se repelem mutuamente. Para que ocorra reação química, é necessário haver quantidade de energia suficiente que supere essa repulsa, promovendo aproximação das moléculas e rompimento das ligações existentes entre os átomos de cada substância, favorecendo a ocorrência de outras liga- ções e, consequentemente, síntese de um novo composto. Portanto, em termos técnicos, é possível afirmar que uma reação química ocorre quando a energia cinética supera a energia de ativação e que catalisadores, por terem a capacidade de diminuir a energia de ativação, colaboram para o aumento da velocidade de reação(4-7).

Os principais fatores que podem influenciar a razão da decomposição do fármaco são os diferentes potenciais hidrogeniônicos $(\mathrm{pH})$ da solução, a temperatura a que o medicamento é exposto, a concentração do fármaco na solução e a exposição à luz ${ }^{(4)}$.

$\mathrm{O}$ pH é definido como concentração de íons hidrogênio presentes em uma solução, sendo que quando igual ao logaritmo negativo $\left(10^{-7}\right)$ é classificado como neutro, pois permite que os íons hidrogênio sejam neutralizados pelos íons hidroxila ${ }^{(8)}$.

Muitos fármacos são suficientemente estáveis na escala de $\mathrm{pH}$ entre 4 e 8 para serem administrados em período de tempo conveniente, sendo menos lesivos ao endotélio vascular, mesmo de veias periféricas de diminuto calibre. Aqueles formulados em valores de $\mathrm{pH}$ mais ácidos, abaixo de 4, possuem maior tempo de validade da apresentação farmacológica e tal característica é identificada em vários antibióticos, o que acarreta o risco para o desenvolvimento de complicações locais da terapia intravenosa. Vale destacar que a associação de fármacos com valores de $\mathrm{pH}$ distintos podem conduzir à alteração de um dos componentes da mistura na solução, gerando instabilidade medicamentosa e aumentando o risco para a ocorrência da incompatibilidade ${ }^{(4)}$.

Considerando que as substâncias mais utilizadas como diluentes para infusão intravenosa de fármacos e eletrólitos são o cloreto de sódio a 0,9\% em água (SF) que apresenta valor médio de $\mathrm{pH}$ de 6,33 e a glicose a $5 \%$ em água (SG), com $\mathrm{pH}$ médio de 6,24 , valores estes aferidos durante a realização desta pesquisa, seria interessante investigar o comportamento ácido-básico de fármacos diluídos nestas soluções, pois é importante que soluções administradas por via IV não apresentem valores extremos de $\mathrm{pH}$, para evitar complicações da terapia intravenosa, como flebite e infiltração(9).

A flebite pode ser classificada em química, mecânica ou infecciosa. A osmolaridade elevada e os extremos de $\mathrm{pH}$ da solução são os principais fatores para o desenvolvimento de flebite química, desencadeando assim, processo inflamatório na túnica íntima da veia. A infiltração é definida como a administração acidental de soluções ou fármacos não vesicantes no espaço extravascular, podendo ser secundária à ocorrência de flebite. A composição e a dosagem do medicamento, a reconstituição e a diluição inadequadas, a mistura de fármacos incompatí- 
veis e a infusão rápida, além de outros fatores, podem resultar em flebite e infiltração ${ }^{(9-11)}$.

Este estudo objetiva analisar os valores de $\mathrm{pH}$, em momentos distintos da infusão de cinco antibióticos frequentemente utilizados em uma unidade de cirurgia pediátrica não climatizada de um hospital universitário da cidade de São Paulo, e tem como hipótese que os fatores diluição e tempo de exposição a condições ambientais resultam em $\mathrm{pH}$ de antibióticos de administração IV diferente do identificado em situação ambiental controlada, a fim de obter subsídios que evidenciem questões de relevância clínica para a enfermagem na realização da terapia IV.

\section{OBJETIVOS}

Medir o potencial de hidrogênio $(\mathrm{pH})$ de antibióticos, em diferentes momentos após a reconstituição e diluição para administração IV em crianças, e expostos a condições ambientais de uma unidade de cirurgia pediátrica; comparar os valores de $\mathrm{pH}$ obtidos com os encontrados em situação laboratorial controlada; identificar os antimicrobianos que possuem valores de $\mathrm{pH}$ que poderiam constituir fator de risco para complicações locais da terapia IV.

\section{MÉTODO}

Após aprovação pelo comitê de ética em pesquisa (no. 1458/06), foi realizado estudo experimental sobre o pH de antibióticos, imediatamente após a reconstituição e diluição e em diferentes momentos que simulam as técnicas de infusão empregadas na prática clínica de enfermagem.

A amostra foi composta por 186 valores de $\mathrm{pH}$ de cinco antimicrobianos, que estão entre os mais utilizados na unidade hospitalar na qual foi realizada esta pesquisa: ceftriaxona sódica, cloridrato de vancomicina, metronidazol, penicilina $\mathrm{G}$ potássica e sulfato de amicacina.

Os experimentos foram realizados três vezes, distribuídos nos dias I, II e III, segundo randomização dos períodos matutino e vespertino.

O preparo dos antimicrobianos e a aferição do pH logo após a reconstituição foram realizados na sala para preparo de medicamentos. A exposição das soluções medicamentosas para infusão IV e a aferição do pH foram realizadas em um quarto destinado a internação das crianças. Ambas as áreas de exposição das soluções eram iluminadas por lâmpadas fluorescentes, sendo este experimento realizado em ambiente hospitalar caracterizado como experimento 1 (E1).

As medidas finais de $\mathrm{pH}$ de um estudo anterior realizado em laboratório com os mesmos antimicrobianos e denominado experimento 2 (E2), desenvolvido em ambiente sem a influência da luz, e climatizado, para obter temperatura de $22^{\circ} \mathrm{C}$, com materiais e equipamentos que caracterizassem condição adequada para a manutenção da esta- bilidade farmacológica, foram utilizadas como valores de referência para comparação da influência das situações de estudo no $\mathrm{pH}$ dos fármacos investigados ${ }^{(11)}$. Os mesmos materiais e técnicas foram empregados no E1 e no E2.

Os valores do $\mathrm{pH}$ foram mensurados em diferentes momentos: imediatamente após a reconstituição em água destilada ou da solução na forma de apresentação líquida; 24 horas após a reconstituição sob refrigeração; t0 - imediatamente após a diluição; t1 - cinco minutos após a diluição; t2 - 30 minutos após a diluição; t3 - 60 minutos após a diluição; t4 - 120 minutos após a diluição; t5 -24 horas após a diluição.

Os fármacos foram reconstituídos com água destilada e diluídos com SF e SG, de acordo com o protocolo de conduta de enfermagem pediátrica para a administração de antimicrobianos do hospital universitário em estudo. Após a reconstituição, a ceftriaxona sódica, o cloridrato de vancomicina e a penicilina $\mathrm{G}$ potássica obtiveram as seguintes concentrações, respectivamente: $100 \mathrm{mg} / \mathrm{ml}$, $100 \mathrm{mg} / \mathrm{ml}$ e $500.000 \mathrm{U} / \mathrm{ml}$. O sulfato de amicacina e o metronidazol não sofreram reconstituição e foram utilizados nas concentrações de $250 \mathrm{mg} / \mathrm{ml}$ e $5 \mathrm{mg} / \mathrm{ml}$, respectivamente. No entanto, este último não foi diluído, pois, segundo recomendação do fabricante, apresenta concentração ideal para a administração por via IV ${ }^{(12)}$.

Para verificar o $\mathrm{pH}$ após a reconstituição, foi retirada uma amostra do frasco-ampola, outra parte foi diluída e o restante da amostra permaneceu no frasco-ampola para ser refrigerado e, posteriormente, permitir a mensuração do $\mathrm{pH}$ da solução submetida a 24 horas de refrigeração.

A concentração final para cada fármaco, após a diluição, foi de $20 \mathrm{mg} / \mathrm{ml}$ para a ceftriaxona sódica; $5 \mathrm{mg} / \mathrm{ml}$ para o cloridrato de vancomicina, metronidazol e sulfato de amicacina; e $50000 \mathrm{U} / \mathrm{ml}$ para a penicilina $\mathrm{G}$ potássica. O volume final das soluções ficou estabelecido em $20 \mathrm{ml}$ para todos os fármacos, sendo as mesmas diluídas e armazenadas em seringas de $20 \mathrm{ml}$.

Em cada momento de aferição, uma amostra da solução armazenada na seringa foi retirada para ser acondicionada em becker de vidro e permitir a mensuração por meio do medidor de $\mathrm{pH}$ digital modelo ExStik ${ }^{\mathrm{TM}} \mathrm{PH} 100$ da marca Extech $^{\circledR}$ (EUA).

A intensidade luminosa do ambiente, em Lux (Ix), foi avaliada com luxímetro digital portátil modelo LD-510 e o controle da temperatura, realizado por meio de dois termômetros digitais, modelo 7429, da marca TFA ${ }^{\circledR}$ Dostmann (Reicholzhein, Baden-Wurttemberg, Alemanha). A temperatura e luminosidade foram verificadas em $t 1, t 3$, t4, t5 e em 24 horas de refrigeração; porém, neste último momento somente foi verificada a temperatura, pois no interior do refrigerador não há luminosidade constante.

Os valores de $\mathrm{pH}$ são apresentados em tabelas e figuras e descritos estatisticamente segundo valores máximo (máx), mínimo (mín), média e desvio padrão (mdp) para 
cada situação estudada. A estatística inferencial foi realizada pelo teste $t$ de Student entre as médias de $\mathrm{pH}$ dos grupos controle e experimento, considerando nível de significância de 5\%.

\section{RESULTADOS}

Foram realizadas 186 medidas de $\mathrm{pH}$, sendo 42 (22,58\%) da ceftriaxona sódica, $42(22,58 \%)$ do cloridrato de vancomicina, 42 (22,58\%) da penicilina G potássica, 39 (20,97\%) do sulfato de amicacina e $21(11,29 \%)$ do metronidazol.

Tanto a temperatura como a iluminância apresentaram grande variação durante os experimentos, sendo que a temperatura ambiental média nos três dias de experimento foi de $26,34^{\circ} \mathrm{C}$, variando de $19,10^{\circ} \mathrm{C}$ a $33,30^{\circ} \mathrm{C}$, demonstrando a condição ambiental de unidade hospitalar não climatizada de um país tropical, representando valores maiores que o recomendado para a exposição de medicamentos durante a sua administração $\left(22^{\circ} \mathrm{C}\right)$. $\mathrm{O}$ refrigerador em que os medicamentos foram armazenados apresentou média de temperatura de $7,30^{\circ} \mathrm{C}$, variando de $2,70^{\circ} \mathrm{C}$ a $13^{\circ} \mathrm{C}$.

Após a reconstituição do antimicrobiano ou abertura do frasco ou ampola, os fármacos menos ácidos foram a ceftriaxona sódica e a penicilina $\mathrm{G}$ potássica, e os que apresentaram valores de $\mathrm{pH}$ mais ácidos foram o sulfato de amicacina e cloridrato de vancomicina. Considerando os três dias de experimentos, todos os antimicrobianos apresentaram desvio padrão menor que 0,24 , para mais ou para menos. O fármaco com maior variação foi o sulfato de amicacina e o com menor, o metronidazol (Tabela 1).

Tabela 1 - Valores e variação do pH dos antimicrobianos após reconstituição ou da apresentação na forma líquida, e armazenados sob refrigeração - São Paulo, 2009

\begin{tabular}{lcccc}
\hline Antimicrobiano & \multicolumn{1}{c}{$\begin{array}{c}\text { pH após } \\
\text { reconstituição ou } \\
\text { da apresentação } \\
\text { líquida }\end{array}$} & $\begin{array}{c}\text { pH após } \\
\text { refrigeração por } \\
\mathbf{2 4} \text { horas }\end{array}$ \\
\cline { 2 - 5 } & Média & $\begin{array}{c}\text { Desvio } \\
\text { Padrão }\end{array}$ & Média & $\begin{array}{c}\text { Desvio } \\
\text { Padrão }\end{array}$ \\
\hline Ceftriaxona Sódica & 6,60 & 0,12 & 6,61 & 0,07 \\
Cloridrato de & 3,48 & 0,12 & 3,66 & 0,20 \\
Vancomicina & & & & \\
Metronidazol & 4,96 & 0,02 & - & - \\
Penicilina G potássica & 6,75 & 0,09 & 6,60 & 0,18 \\
Sulfato de Amicacina & 4,47 & 0,23 & - & - \\
\hline
\end{tabular}

Verifica-se que mesmo após o armazenamento por 24 horas, não houve mudança significativa nos valores de $\mathrm{pH}$ dos antimicrobianos, se comparados com a média dos valores no momento logo após reconstituição (Tabela 1), destacando-se que todos estes medicamentos podem ser mantidos sob refrigeração por este período de tempo, segundo recomendações do fabricante.

A Figura 1 permite a visualização dos valores de $\mathrm{pH}$ do metronidazol e dos fármacos diluídos em SF e expostos a condições ambientais diferentes (E1 e E2).

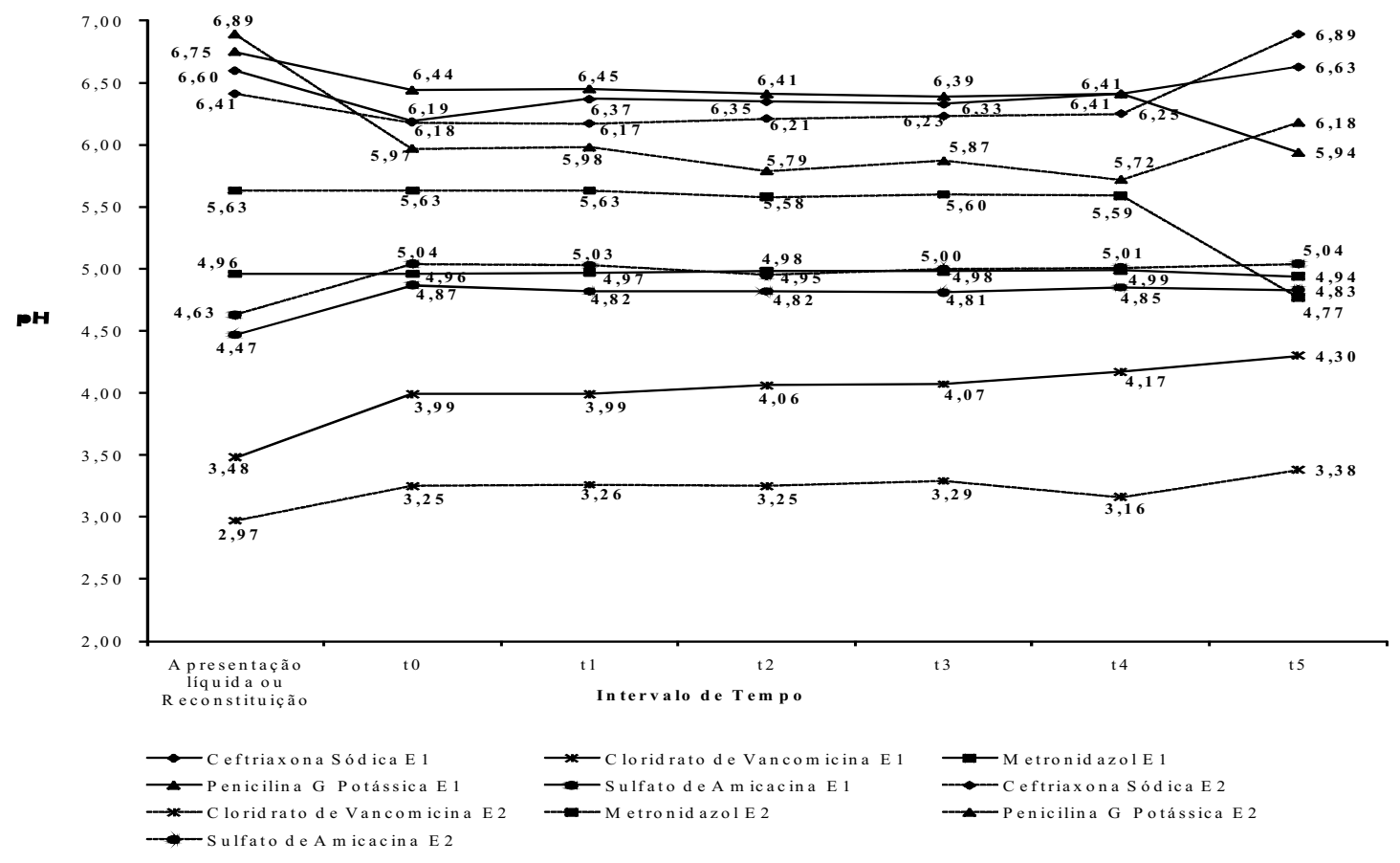

Figura 1 - Valores de $\mathrm{pH}$ dos antimicrobianos diluídos em SF e do metronidazol, segundo intervalo de tempo, de acordo com Experimento 1 e Experimento 2 - São Paulo, 2009 
Em ambos os experimentos, a ceftriaxona sódica e a penicilina $\mathrm{G}$ potássica tornaram-se mais ácidas após serem diluídas em SF enquanto os antimicrobianos cloridrato de vancomicina e sulfato de amicacina tornaram-se menos ácidos (Figura 1).

Entretanto, esses dois últimos foram os fármacos que resultaram nos menores valores de $\mathrm{pH}$, o cloridrato de vancomicina apresentou média de 4,09+0,13 e 3,27+0,07, respectivamente em E1 e E2, e o sulfato de amicacina, valores médios de 4,83+0,20 e 5,01+0,03, respectivamente em E1 e E2 (Tabela 2).

Em 24 horas de exposição, a ceftriaxona sódica e o cloridrato de vancomicina obtiveram $\mathrm{pH}$ maior do que nos demais intervalos de tempo. Já a penicilina $G$ potássica tornou-se mais ácida ao longo do tempo em E1 e menos ácida em E2. O sulfato de amicacina, por sua vez, manteve seu comportamento ácido-básico sem alterações consideráveis no decorrer do experimento e o cloridrato de vancomicina apresentou tendência ao aumento de $\mathrm{pH}$ ao longo do tempo, em ambos os experimentos. O metronidazol revelou variação de $\mathrm{pH}$ diminuta, delineando curva estável, ao longo de todo tempo de exposição, exceto em 24 horas em E2 (Figura 1).

Tabela 2 - Valores de $\mathrm{pH}$ dos antimicrobianos, segundo o diluente, e do metronidazol, de acordo com Experimento 1 e o Experimento 2 - São Paulo, 2009

\begin{tabular}{|c|c|c|c|c|c|c|c|c|c|c|}
\hline \multirow[b]{2}{*}{ Antimicrobianos } & \multirow[b]{2}{*}{ Diluentes } & \multicolumn{4}{|c|}{ pH E1 } & \multicolumn{4}{|c|}{ pH E2 } & \multirow[b]{2}{*}{$\mathrm{p}^{*}$} \\
\hline & & Min & Máx & Média & $\begin{array}{l}\text { Desvio } \\
\text { Padrão }\end{array}$ & Min & Máx & Média & $\begin{array}{l}\text { Desvio } \\
\text { Padrão }\end{array}$ & \\
\hline Celtriaxona & $\mathrm{SF}$ & 5,85 & 6,66 & 6,38 & 0,17 & 6,17 & 6,89 & 6,32 & 0,28 & 0,3241 \\
\hline Sódica & SG & 6,25 & 6,75 & 6,49 & 0,11 & 6,26 & 6,94 & 6,63 & 0,25 & 0,0301 \\
\hline Cloridrato de & $\mathrm{SF}$ & 3,95 & 4,39 & 4,09 & 0,13 & 3,16 & 3,38 & 3,27 & 0,07 & $<0,0001$ \\
\hline Vancomicina & SG & 3,76 & 4,1 & 3,96 & 0,08 & 3,27 & 3,65 & 3,39 & 0,15 & $<0,0001$ \\
\hline Metronidazol & - & 4,91 & 5,04 & 4,97 & 0,03 & 4,77 & 5,63 & 5,47 & 0,34 & 0,0083 \\
\hline Penicilina G & SF & 5,89 & 6,52 & 6,34 & 0,2 & 5,72 & 6,18 & 5,92 & 0,16 & 0,0002 \\
\hline Potássica & SG & 6,28 & 6,77 & 6,6 & 0,15 & 6,42 & 6,74 & 6,64 & 0,12 & 0,2829 \\
\hline Sulfato de & $\mathrm{SF}$ & 4,54 & 5,07 & 4,83 & 0,02 & 4,95 & 5,04 & 5,01 & 0,03 & 0,001 \\
\hline Amicacina & SG & 4,59 & 5,06 & 4,87 & 0,19 & 5,02 & 5,09 & 5,06 & 0,02 & 0,0138 \\
\hline
\end{tabular}

Legenda: SF- cloreto de sódio 0,9\%; SG- Soro Glicosado a 5\%; E1 - Experimento 1; E2 - Experimento 2 . $^{*}$ Valor obtido pela aplicação do teste t.

Dos medicamentos diluídos em SG, o cloridrato de vancomicina foi o antimicrobiano mais ácido ao longo do tempo, com média de 3,96+0,08 em E1 e 3,39+0,15 em E2.

Já o antimicrobiano menos ácido foi a penicilina $G$ potássica: 6,60+0,15 em E1 e 6,64+0,12 em E2 (Tabela 2).
Na Tabela 2, observa-se que na diluição com SF os fármacos com maior variabilidade nos valores de $\mathrm{pH}$ em E1 foram o sulfato de amicacina $(4,83+0,20)$ e a penicilina $G$ potássica $(6,34+0,20)$. Já em E2, os antimicrobianos com maior variabilidade após o uso deste mesmo diluente foi a ceftriaxona sódica $(6,32+0,28)$. Contudo, todas as soluções de antimicrobianos diluídas em SF apresentaram uma variação menor que 1,0 valor de $\mathrm{pH}$ ao longo do tempo nos dois experimentos.

Ao comparar os experimentos, observa-se que o metronidazol, fármaco comercializado na apresentação líquida e com concentração ideal para administração IV, apresentou maior alteração no valor de $\mathrm{pH}(5,47+0,34)$ ao longo do tempo de exposição no E2 (Tabela 2).

Excetuando-se a ceftriaxona sódica, todos os fármacos diluídos em SF apresentaram diferença significativa entre os valores médios de $\mathrm{pH}$ quando comparados os dois experimentos (Tabela 2). Destaca-se que os frascos de medicamentos utilizados em E1 e E2 não foram os mesmos, ou seja, o que pode ter gerado valores de $\mathrm{pH}$ distintos ao longo do tempo pode ter sido o $\mathrm{pH}$ inicial diferente para cada frasco e não as condições ambientais, apesar de ser esperado inicialmente, que medicamentos de um mesmo fabricante submetidos a um mesmo procedimento de análise não apresentassem as variações identificadas. 


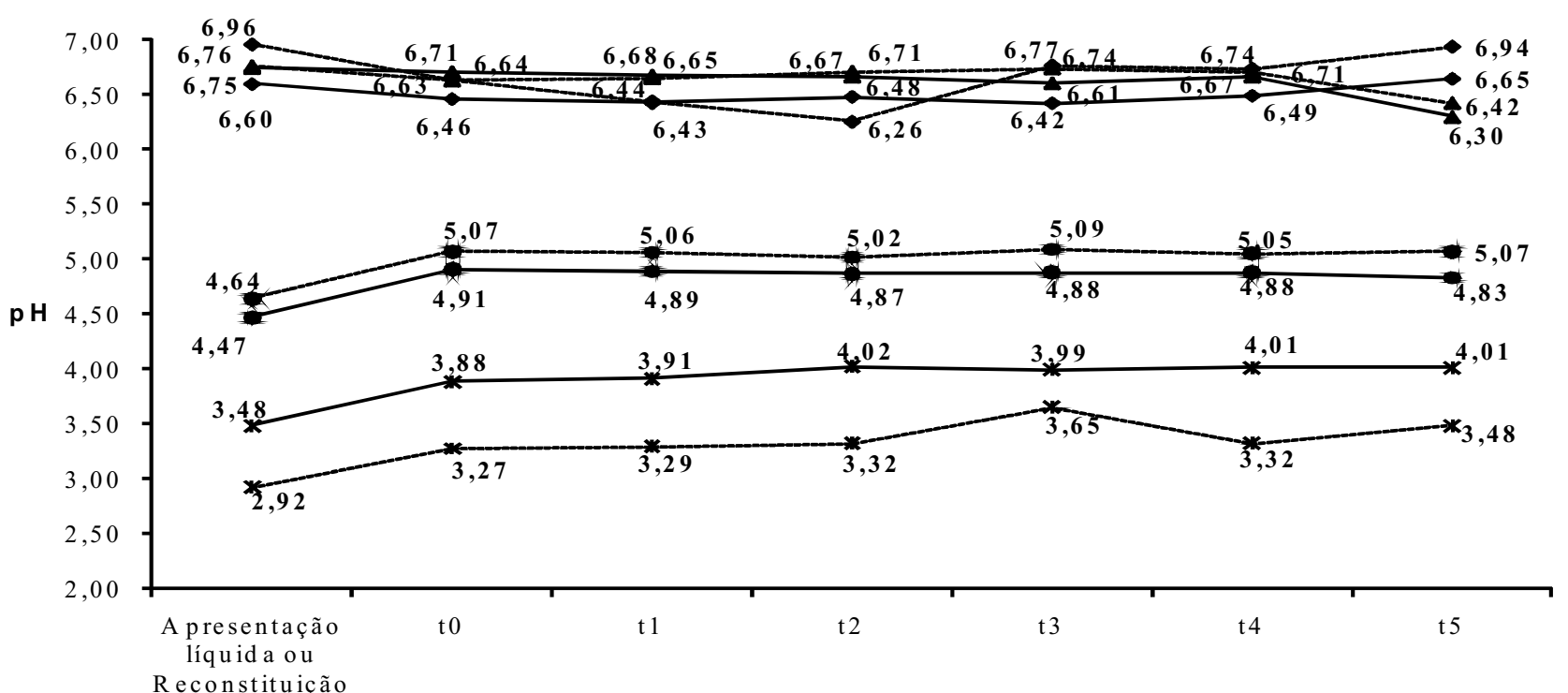

Intervalo de Tem po
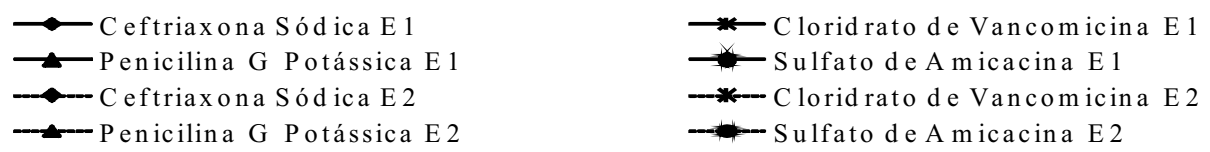

Figura 2 - Valores de $\mathrm{pH}$ dos antimicrobianos diluídos em soro glicosado a 5\%, segundo intervalo de tempo, de acordo com Experimento 1 e Experimento 2 - São Paulo, 2009

Observa-se por meio da Figura 2 que, em ambos os experimentos, o sulfato de amicacina apresentou variação de $\mathrm{pH}$ diminuta ao longo do tempo. O cloridrato de vancomicina demonstrou-se estável em E1, no entanto, em E2 revelou aumento de $\mathrm{pH}$ em $\mathrm{t} 3$ e decréscimo em seguida. Já a penicilina $\mathrm{G}$ potássica, embora tenha se mantido bastante estável ao longo do tempo, apresentou queda de $\mathrm{pH}$ em 24 horas de exposição em E1 e em E2. Em relação à ceftriaxona, observa-se que, apesar de em E1 demonstrar comportamento estável ao longo do tempo com aumento de pH em 24 horas de exposição, em E2 ocorreu acidificação até o tempo $t 2$, a partir do qual se iniciou aumento do valor de $\mathrm{pH}$, com valor máximo em $\mathrm{t} 5$.

Todas as soluções de antimicrobianos diluídas em SG apresentaram variação menor que 1,0 valor de $\mathrm{pH}$ ao longo do tempo nos dois experimentos.

Excetuando-se a penicilina $\mathrm{G}$ potássica, todos os fármacos diluídos em SG apresentaram $\mathrm{pH}$ diferente nos dois experimentos (Tabela 2). Semelhantemente ao ocorrido nos experimentos em que os medicamentos foram diluídos em SF, a diferença entre as médias foi identificada já nos valores iniciais, evidenciando $\mathrm{pH}$ distintos, em frascos ou ampolas diferentes de um mesmo fármaco de determinado fabricante.

Comparando os resultados conforme o diluente, observa-se que o cloridrato de vancomicina tende a se tornar mais ácido na diluição com SG. Já a ceftriaxona sódica, a penicilina $\mathrm{G}$ potássica e o sulfato de amicacina tiveram comportamento mais ácido com o SF como diluente. No E2 todos os fármacos foram mais ácidos quando diluídos com o SF (Tabela 2).

\section{DISCUSSÃO}

A administração de fármacos e soluções por via IV na criança constitui desafio para prática de enfermagem baseada em evidências e para a promoção de intervenções seguras, devido à falta de fármacos com apresentação para esta faixa etária, bem como de estudos sobre a temática ou respaldo da indústria farmacêutica que permitam ao enfermeiro analisar a adequação científica das atividades realizadas rotineiramente na prática assistencial. Assim, faz-se importante que a enfermagem investigue as bases que fundamentam a sua prática na administração da terapia medicamentosa, promovendo o avanço da ciência e das intervenções de enfermagem prestadas ao paciente.

Diante da problemática dos fármacos não serem comercializados com apresentação específica para faixa etária pediátrica, os profissionais reconstituem os antimicrobianos, os armazenam em refrigeradores, posteriormente realizam a diluição em volumes e soluções próprias para infusão IV e os administram em maior período de tempo para controlar fatores adversos deletérios à população infanti(l(2). Por esses motivos, muitos fármacos acondicionados em frasco-ampola são reaproveitados em serviços de saúde evitando, desta forma, o desperdício de antimicrobianos que são medicamentos de alto custo, contribuindo 
para a redução dos gastos da instituição. No entanto, esta reutilização pode comprometer a qualidade e a segurança da terapêutica IV ${ }^{(13)}$.

Surgem, assim, questionamentos quanto às possíveis alterações do comportamento químico das soluções após sua refrigeração, diluição e quanto à ação de catalisadores como temperatura e luz, por longos períodos de infusão em condições ambientais de unidades hospitalares não climatizadas.

A temperatura, tanto in vitro como in vivo, está relacionada de forma diretamente proporcional à quantidade de movimento vibratório aleatório dos átomos que compõem as moléculas de um dado sistema, tendo a capacidade de influenciar a velocidade de reação química ao promover energia cinética superior à energia de ativação. De modo geral, a cada aumento em $10^{\circ} \mathrm{C}$ de uma solução, a velocidade de reação pode duplicar e até quintuplicar ${ }^{(14)}$.

Para o armazenamento de frascos em refrigeradores, deve-se manter a temperatura de $4^{\circ} \mathrm{C}$ a $8^{\circ} \mathrm{C}$ a fim de conservar a estabilidade farmacológica(15). Na presente investigação a temperatura mínima do refrigerador foi $2,7^{\circ} \mathrm{C}$ e máxima $13^{\circ} \mathrm{C}$. Esta variação não poderia ocorrer a fim de manter as características desejadas dos medicamentos utilizados, porém a frequente abertura do refrigerador impede que a temperatura permaneça em níveis mais seguros. Quanto às variações de $\mathrm{pH}$, esta situação não causou influência significativa, contudo não permite analisar aspectos outros referentes a qualidade terapêutica das soluções estudadas. A enfermagem ao assumir a responsabilidade pela manipulação de medicamentos em unidades hospitalares deve requerer estrutura que permita realizar práticas seguras.

$\mathrm{O}$ aumento da temperatura do ambiente influencia a degradação das soluções, alterando o pH e consequentemente levando à instabilidade do fármaco. A penicilina $G$ potássica e as cefalosporinas são descritas na literatura como lábeis ao calor ${ }^{(4)}$. A penicilina $G$ potássica diluída em SF apresentou o maior desvio padrão $(0,20)$ o que evidencia característica mais lábil deste fármaco.

Experimento realizado ${ }^{(16)}$ evidenciou que esse antimicrobiano, na concentração de $5000 \mathrm{U} / \mathrm{mL}$, apresentou mínima variação de $\mathrm{pH}$ nas temperaturas de $4^{\circ} \mathrm{C}, 25^{\circ} \mathrm{C}$ e $37^{\circ} \mathrm{C}$, em SF $(5,89$ a 6,19$)$ e SG $(4,82$ a 6,39$)$. Segundo a presente pesquisa a penicilina $\mathrm{G}$ potássica diluída em $\mathrm{SF}$ apresentou média de $\mathrm{pH}$ de 6,34 e em SG de 6,60, permitindo observar que apresentou valor médio mais elevado se comparado com o estudo supra citado em ambos os diluentes. No E2 este fármaco ao ser diluído em SF apresentou valores muito próximos aos encontrados no E1, principalmente quando utilizado como diluente SG. Ressalta-se que a concentração da penicilina, no estudo de Wyatt, foi dez vezes menor que a concentração obtida com o mesmo antimicrobiano no E1 e no E2.

Ademais, pode-se verificar por meio da literatura, que a penicilina $\mathrm{G}$ potássica é estável por período inferior a 24 horas $^{(17)}$. Neste estudo, o fármaco apresentou pequena variação nos valores de $\mathrm{pH}$ até 24 horas após exposição à condição ambiental.

Pesquisa experimental identificou que a ceftriaxona sódica em água destilada $(100 \mathrm{mg} / \mathrm{mL})$ e armazenada em seringas de polipropileno mantinha-se estável por 40 dias em temperatura igual a $4^{\circ} \mathrm{C}$, e por cinco dias a $20^{\circ} \mathrm{C}$, com alterações na coloração da solução. Isto foi associado à mudança mínima no valor do $\mathrm{pH}(7,07$ a 7,13) entre 24 a 120 horas $^{(18)}$. Os valores de $\mathrm{pH}$ foram maiores que os encontrados em nosso estudo devido à diferença de concentração das soluções e aos diluentes utilizados, que são mais ácidos do que a água destilada, que por sua vez constitui fator de risco para ocorrência de complicações.

Ao comparar os valores obtidos no E1 e E2, observase que a ceftriaxona sódica apresentou maior variação quando exposta a condição ambiental de $22^{\circ} \mathrm{C}$ e sem luz, independentemente do diluente utilizado, o que contradiz a informação de que este fármaco é lábil ao calor. A ceftriaxona sódica não apresentou variações significativas uma vez que ao ser diluída em SF, apresentou média de $6,38(+0,17)$ e em SG de $6,49(+0,11)$.

Quanto à luminosidade, sabe-se que ativa reações de fotodegradação, como a oxidação e hidrólise. Assim, quanto mais intensa for uma fonte de luz e ou quanto mais próximo o fármaco fotossensível estiver desta fonte, maior será a taxa e o grau de degradação ${ }^{(4)}$. A luz, radiação eletromagnética, se propaga no espaço transportando energia que pode, por meio da fotólise, decompor uma molécula pelo desdobramento ou rearranjo de suas ligações químicas. O comprimento de onda é inversamente proporcional à sua energia e assim, a fotodegradação do fármaco aumenta, respectivamente, na seguinte sequência: iluminação do tipo incandescente, fluorescente, solar e de raios ultravioleta ${ }^{(14)}$.

O metronidazol é descrito como fármaco sensível à luminosidade durante o armazenamento e administração. Contudo, na prática clínica, durante a sua administração por via IV, a proteção da luz do ambiente não é uma preocupação ${ }^{(12)}$. Os experimentos realizados na presente pesquisa, mostram que não houve alterações significativas no valor de $\mathrm{pH}$ deste fármaco, que apresentou menor desvio padrão $(0,03)$ em comparação com os demais fármacos investigados, e média de $\mathrm{pH}$ de 4,97, mesmo sendo administrado em unidade não climatizada e sem utilização de sistemas de infusão com fotoproteção.

Assim, observa-se que mesmo quando exposto a condição ambiental com luminosidade natural manteve seu comportamento ácido e estável (E1).

Com relação às complicações da terapia IV, um estudo clínico nacional realizado em crianças revelou que 4,7\% dos cateteres IV periféricos foram removidos devido à flebite e 55,3\% devido à infiltração ${ }^{(19)}$. A irritação significativa do endotélio, causada pela composição química da solu- 
ção, pH ou osmolaridade, pode danificar a camada íntima da veia e caracterizar a ocorrência de flebite, com possibilidade de ocorrência de infiltração secundária ${ }^{(10)}$.

A faixa de risco para as complicações da terapia IV corresponde aos valores de $\mathrm{pH}$ inferiores a 5,5 e superiores a $8,0^{(9)}$. A antibioticoterapia aumenta o risco do desenvolvimento de flebite ${ }^{(20)}$, devido principalmente ao tempo de exposição do endotélio a esta substância. Pesquisa identificou que a incidência desta complicação quando da administração de antibióticos foi de $18,5 \%$, enquanto que sem a utilização deste tipo de fármaco foi de $8,8 \%{ }^{(21)}$. Tal resultado pode ter relação com o pH eminentemente ácido destes fármacos.

As cefalosporinas, penicilinas e a vancomicina, são considerados antimicrobianos irritantes ao endotélio vascular $^{(22)}$. Adiciona-se que soluções constituídas por glicose aumentam o risco de ocorrência de flebite, em comparação com o $\mathrm{SF}^{(20)}$. No presente estudo, nota-se que o cloridrato de vancomicina, o sulfato de amicacina, independentemente do diluente utilizado, e o metronidazol, constituem fator de risco para o desenvolvimento de complicações locais da terapia IV.

Alguns medicamentos são instáveis em soluções ácidas ( $\mathrm{pH}$ menor que 4) ou alcalinas ( $\mathrm{pH}$ maior que 8), dentre estes fármacos destacam-se alguns antimicrobianos ${ }^{(7)}$. Por este motivo, é de extrema importância a análise prévia do $\mathrm{pH}$ dos componentes de uma mistura, a fim de evitar prejuízos terapêuticos e ou complicações ${ }^{(4)}$.

Neste estudo, o único antimicrobiano que apresentou valores de $\mathrm{pH}$ extremos foi o cloridrato de vancomicina diluído em SF $(3,95$ a 4,39) e SG $(3,76$ a 4,10). Ao comparar estes dados com os obtidos no $E 2$ nota-se que os valores foram ainda menores (3,16 a 3,38 em SF e 3,27 a 3,65 em SG).

É possível observar que ao comparar os dois experimentos (E1 e E2) os antimicrobianos que apresentaram variação estatisticamente significante foram o cloridrato de vancomicina, penicilina $\mathrm{G}$ potássica e sulfato de amicacina, quando utilizado o diluente $S F$, e a ceftriaxona sódica, cloridrato de vancomicina e o sulfato de amicacina, diluídos em SG. Assim, os antibióticos que apresentaram alterações significativas não são exatamente os mesmos para ambos os diluentes. É possível inferir que pode existir diferença entre as médias porque os frascos de medicamentos utilizados no E1 não foram os mesmos do E2, ou seja, o que pode ter levado a valores de $\mathrm{pH}$ distintos ao longo do tempo foi o $\mathrm{pH}$ inicial diferente para cada frasco e não as condições ambientais.

Tal constatação é relevante, pois configura diferenças não esperadas entre medicamentos de um mesmo fabricante. Ressalta-se que grande parte dos medicamentos utilizados neste estudo são classificados como off label e fármacos genéricos.

Pode-se observar por meio desta pesquisa, que todos os antimicrobianos apresentaram variação menor que 1,0 valor de $\mathrm{pH}$ ao longo do tempo de exposição às condições ambien- tais. Se tal variação é capaz de alterar ou não as propriedades farmacológicas das substâncias estudadas, constitui objeto de estudo para futuras investigações sobre a temática com utilização de outras metodologias de análise de estabilidade farmacológica como cromatografia líquida de alta performance (mais conhecida como HPLC - High Performance Liquid Cromatography). Consiste em método físico-químico que separa líquidos de mistura em seus componentes individuais, identificando e quantificando os produtos finais da mistura, sendo esses dados registrados em um cromatograma ${ }^{(14)}$.

Entre as limitações do estudo destaca-se que não há como determinar possíveis alterações nas propriedades terapêuticas dos fármacos apenas com a medida de $\mathrm{pH}$ e o fato de no $\mathrm{E} 2$ as medidas de $\mathrm{pH}$ terem sido realizadas uma única vez em cada experimento. Além disso, foram comparadas as médias de $\mathrm{pH}$ nos E1 e E2 que foram obtidas de frascos diferentes e cujos valores iniciais já eram distintos, o que pode ter comprometido a comparação final.

No entanto, os resultados obtidos revelam a necessidade de repensar a prática clínica e desenvolver estudos colaborativos entre a enfermagem e a farmacologia com vistas a promover práticas de enfermagem baseadas em evidências. Despertam à reflexão do enfermeiro, ao utilizar um medicamento em sua prática clínica, quanto à necessidade de realizar análise crítica do respaldo fornecido pela indústria para a administração segura de medicamentos por via IV. Também demonstram que, mesmo após a aplicação de protocolo de diluição rotineiramente utilizado para infusão de antimicrobianos em crianças, o pH permanece próximo ao obtido na apresentação farmacológica, mantendo o risco para o desenvolvimento das complicações da terapia intravenosa que se pretende prevenir com a diluição, a flebite e a infiltração, sendo este fato identificado em todas as medidas, independentemente do diluente empregado.

\section{CONCLUSÃO}

Não foram observados valores discrepantes de $\mathrm{pH}$ da apresentação farmacológica ou após reconstituição. Destacam-se a ceftriaxona sódica, penicilina $\mathrm{G}$ potássica e sulfato de amicacina, como os antimicrobianos que sofreram maior variação no valor de $\mathrm{pH}$ ao longo do tempo, independentemente do diluente utilizado. Não foram identificadas variações maiores que 1,0 valor de $\mathrm{pH}$ ou que caracterizassem alterações do comportamento de ácido para básico, ou vice-versa, das soluções.

Quando comparados com estudo similar, observouse que os fármacos que apresentaram valores de $\mathrm{pH}$ com diferença estatisticamente significante foram o cloridrato de vancomicina, a penicilina $G$ potássica e o sulfato de amicacina diluídos em SF, e o metronidazol. Ao utilizar o diluente SG, foram os antimicrobianos ceftriaxona sódica, cloridrato de vancomicina e sulfato de amicacina que apresentaram diferença significante. 
Alguns dos valores de $\mathrm{pH}$ obtidos constituem fator de risco para o desenvolvimento de complicações locais da terapia IV, em especial o cloridrato de vancomicina, o sulfato de amicacina e o metronidazol, não estando relacionados ao diluente utilizado.

Esta investigação desperta para a necessidade de aprofundamento do conhecimento por meio da realização de

\section{REFERÊNCIAS}

1. Kuhn M. Pharmacotherapeutics: a nursing process approach. 4th ed. Philadelphia: FA Davis; 1998.

2. Peterlini MAS, Chaud MN, Pedreira MLG. Órfãos de terapia medicamentosa: a administração de medicamentos por via intravenosa em crianças hospitalizadas. Rev Latino Am Enferm. 2003;11(1):88-95.

3. Pedreira MLG, Chaud MN. Terapia intravenosa em pediatria: subsídios para a prática de enfermagem. Acta Paul Enferm. 2004;17(2):222-8.

4. Trissel LA. Drug stability and compatibility issues in drug delivery. In: Trissel LA. Handbook of injectable drugs. 11th ed. Bethesda: American Society of Health-System Pharmacistis; 2001.

5. Reis LL, Peterlini MAS, Pedreira MLG. Potencial hidrogeniônico de soluções de cloridrato de dobutamina expostas a condições ambientais de unidades de cuidados intensivos neonatais. J Pediatr. 2009;85(6):553-6.

6. Stella VJ. Chemical and physical bases determinin the instability and incompatibility of formulated injectable drugs. J Parent Sci Technol. 1986;40(4):142-63.

7. Newton DW. Physicochemical determinants of incompatibility and instability in injectable drugs solutions and admixtures. Am J Hosp Pharm. 1978;35(10):1213-22.

8. Weinstein SM. Plumer's principles and practice of intravenous therapy. 6th ed. Philadelphia: Lippincott; 1997.

9. Phillips LD. Manual de terapia intravenosa. 2a ed. Porto Alegre: Artmed; 2001.

10. Pettit J. Assessment of an infant with a peripheral intravenous device. Adv Neonatal Care. 2003;5(5):230-40.

11. Crepaldi RMC, Monteiro C, Peterlini MAS, Pedreira MLG. Hydrogen-ion potential of antibiotics according to the environment factors temperature and luminosity. Rev Latino Am Enferm. 2010;18(2):278-86.

12. Micromedex ${ }^{\circledR}$ Healthcare Series [Internet]. New York: Thomson Reuters; 2009 [cited 2009 Aug 23]. Available from: https://www.thomsonhc.com/hcs/librarian/CS/B466C4/ PFActionld/pf.HomePage/ssl/true estudos sobre essa temática, possibilitando ao enfermeiro embasamento e respaldo científico no planejamento e na implementação de cuidados, resultando em um atendimento mais qualificado e seguro, uma vez que este profissional é fundamental na administração da terapia medicamentosa, em especial em crianças, que exigem cuidados específicos para garantir a eficácia desta terapêutica.

13. Souza MCP, Goulart MA, Rosado V, Reis AMM. Utilização de medicamentos parenterais em frascos-ampola em uma unidade pediátrica de um hospital universitário. Rev Esc Enferm USP. 2008;42(4):715-22.

14. Reis LL. Potencial hidrogeniônico de soluções de cloridrato de dobutamina expostas a condições ambientais de unidades de cuidados intensivos neonatais [dissertação]. São Paulo: Universidade Federal de São Paulo; 2007.

15. Telles PCPF, Cassiani SHB. Administração de medicamentos: aquisição de conhecimentos e habilidades requeridas por um grupo de enfermeiros. Rev Latino Am Enferm. 2004;12(3):533-40.

16. Wyatt RG, Okamoto GA, Feigin RD. Stability of antibiotics in parenteral solutions. Pediatrics. 1972;49(1):22-9.

17. Gilbert DN, Dworkin RJ, Raber SR, Leggett JE. Outpatient parenteral antimicrobial-drug therapy. N Engl J Med. 1997;337(12):829-39.

18. Plumridge RJ, Rieck AM, Annus TP, Langton SR. Stability of ceftriaxone sodium in polypropylene syringes at $-20,4$, and 20 degrees C. Am J Health Syst Pharm. 1996;53(19):2320-3.

19. Machado AF, Pedreira MLG, Chaud MN. Eventos adversos relacionados ao uso de cateteres intravenosos periféricos em crianças de acordo com tipos de curativos. Rev Latino Am Enferm. 2008;16(3):362-7.

20. Maki DG, Ringer M. Risk factors for infusion-related phlebitis with small peripheral venous catheters: a randomized controlled trial. Ann Intern Med. 1991;114(10):845-54.

21. Lanbeck P, Odenholt I, Paulsen O. Antibiotics differ in their tendency to cause infusion phlebitis: a prospective observational study. Scand J Infect Dis. 2002;34(7):512-9.

22. Turnidge J. Hazards of peripheral intravenous lines. Med J Aust. 1984;141(1):37-40. 